\title{
Fractionating the Rational Brain
}

\author{
Vinod Goel \\ York University, Canada \\ http://www. yorku.ca/vgoel
}

Considerable progress has been made over the past decade in our understanding of the neural basis of logical reasoning. Unsurprisingly these data are telling us that the brain is organized in ways not anticipated by cognitive theory. In particular, they're forcing us to confront the possibility that there may be no unitary reasoning system in the brain (be it mental models or mental logic). Rather, the evidence points to a fractionated system that is dynamically configured in response to certain task and environmental cues. I will review three lines of demarcation including (a) systems for heuristic and formal processes (with evidence for some degree of content specificity in the heuristic system), (b) conflict detection/resolution systems, and (c) systems for dealing with certain and uncertain inferences; and then offer a tentative account of how the systems might interact to facilitate logical reasoning. Sensitivity to data generated by neuroimaging and patient methodologies will move us beyond the sterility of mental models vs. mental logic debate and further the development of cognitive theories of reasoning. 